\title{
A simple polygon-polygon basis method to compare categorical raster maps
}

\author{
Celestino Ordóñez ${ }^{1, a}$, José M. Matías ${ }^{2}$ Javier Taboada $^{1}$ Teresa Rivas $^{1}$ \\ ${ }^{1}$ Dept. Natural Resources and Environmental Engineering, University of Vigo, 36310, Vigo, Spain \\ ${ }^{2}$ Dept. of Statistics, University of Vigo, 36310, Vigo, Spain
}

Received 20 September 2008, Accepted 12 March 2009

\begin{abstract}
In this paper we present a method for comparing categorical raster maps based on comparing categories at the polygon level. In contrast with the most commonly used pixel-level methods of comparison, the proposed method reduces both possible map registration errors and errors resulting from the uncertainty associated with defining the boundaries of each category. The results are presented visually as a map which shows the degree of fit between the compared maps, and analytically as an overall agreement index varying between 0 and 1 and by a fuzzy kappa index.

The methodology is illustrated by two examples: the first compares two synthetic maps which were generated specifically to demonstrate the validity of the method, and the second example analyses the use of the method to detect changes over time in two real land-use maps which were made in different years.
\end{abstract}

Key words: raster model; map comparison; categorical data; fuzzy kappa

\section{Introduction}

Categorical maps are commonly compared to detect differences between maps that have been made by different persons or using different techniques, and also to detect possible errors (Foody [4]). Map comparison is also very often used to determine variations over time-for example, changes in land-use (Wickware and Howarth [14], Singh [12]).

Traditional methods of comparison are based on a pixelby-pixel comparison of each map, with the results displayed as crossed tabulations and statistical indices such as the kappa coefficient of agreement (Rosenfield and Fitzpatrick-Lins [11] ) or the tau coefficient of agreement (Ma and Redmond [7]). However, as other authors have noted (Mas [8], Dai and Khorram [2]), these methods of comparison present some drawbacks. Firstly, they are sensitive to image registration. Secondly, Boolean intersections do not take into account the uncertainty associated with each category and the definition of the boundaries. The kappa index, one of the most commonly used methods for compare categorical maps and evaluating map precision, also presents some problems (Pontius [9]).

Over the last few years researchers have proposed alternative methods of comparing maps in order to address some of the drawbacks associated with these traditional methods. Many of these alternative methods are classed as fuzzy, although few of them are actually based on diffuse logic theory. Fritz and See [5] tackled the problem of comparing maps which have different categories. The comparison of categories is made by using similarity matrices for consultations made with several experts, with findings translated into similarity values ranging between 0 (no similarity) and 1 (total similarity) that are known as fuzzy agreement values. Finally, Hagen et al. [6] used a method of comparing categorical raster maps pixel by pixel which took into account possible errors in space and in the category definition by analyzing the area around each pixel. Matrices of similarity are also defined to take into account the fact that some categories are more similar to each other than others, and to take into account the distance between each cell to neighboring cells in order to establish the degree of similarity between each map for each location; the form of these functions, however, is not specified. The fit between maps is measured by a statistic called fuzzy kappa. Power et al. [10] proposed a method for comparing land-use maps based on formal fuzzy logic theory. In this method linguistic variables and functions of membership are defined which consider the percentage of the area of intersection between polygons of the same category. Comparison is made at the regional level instead of pixel by pixel. They express the overall agreement or estimate of variation between two land-use maps using a scalar between 0 and 1 called a fuzzy global similarity value.

The method we describe in this paper is mainly based on work carried out by Power et al. [10] and Hagen et al. [6].On the one hand, as with the first author, maps are compared at a regional level, in other words at the level of polygons rather than pixels. On the other hand, uncertainty in delimiting categories is taken into account using a simple method based on the distance to boundary cells. The fit between two maps is summarized by a coefficient of similarity. The fuzzy kappa index is also determined

\footnotetext{
${ }^{a}$ Corresponding author: C.Ordóñez cgalan@uvigo.es
} 
and is compared with Boolean kappa.

\section{Methodology}

In the methodology described below, one the two maps to be compared map is considered as the reference map $(A)$ , against which the fit of the other map $(B)$ is determined. Below we describe the steps to follow in obtaining a map and an index of fuzzy agreement between map $A$ and $B$ :

1. The first step is to delimit the polygons that correspond to each category of the legend in each of the two maps to be compared using a reclassification process. In this way, for each original map, a map is generated for each category, as follows:

$$
\begin{array}{ll}
C_{i}^{A} & i: 1, \ldots, r \\
C_{i}^{B} & i: 1, \ldots, r
\end{array}
$$

where the superscript refers to the original map and the subscript refers to the delimited category, and where $r$ is the number of legend categories in both maps. Irrespective of the original legends of the maps that are to be compared, all of the cells of the polygons that are delimited on each reclassified map are assigned the value 1 . The rest of the cells that do not correspond to the category being considered are assigned the value 0 . Thus, for each map that is reclassified from two maps being compared, the following condition is met:

$$
V_{l}^{C_{j}^{A, B}}=\delta_{C_{l}, j}
$$

where $V_{l}^{C_{j}^{A, B}}$ represents the value of the cell which corresponds to a location $l$ in the $j$ th map (corresponding to the $j$ th category) originating from map $A$ or $B, \delta$ is the Kronecker delta, and $C_{l}$ is the category of the original map at location $l$.

By performing local matching on a polygon-bypolygon basis, the problems of a pixel-by-pixel comparison are reduced.

2. For each of the maps obtained in the previous stage from map $A, C_{i}^{A}$, the boundary of each polygon is determined, with the boundary pixels assigned the value 1 and the other pixels assigned the value 0 . We will refer to these maps as $H_{i}^{A} \quad i: 1, \ldots, r$.

3. We then determine the distances from each cell in maps $H_{i}^{A}$ to the boundary cells, to give maps $D_{i}^{A} \quad i: 1, \ldots, r$. Then for each cell of $D_{i}^{A}$ which is situated on the outside of the boundary of each polygon, a function $f$ is applied that decreases with distance; meanwhile, for the cells located inside the boundary of the polygon a function $g$ is applied, which increases with distance. In doing so we are acknowledging that the boundaries of the polygons are not precise, with imprecision increasing from the inside of the polygons to the outside. The resulting maps are named $F_{i}^{A}$ and $G_{i}^{A}$, to indicate that they result from the application of functions $f$ and $g$, respectively.

Functions $f, g: R^{+} \rightarrow[0,1]$ are defined subjectively and may be different for each reclassified map according to the knowledge we have on the precision of the definition of each polygon in map A.

4. In this step, maps $F_{i}^{A}\left(G_{i}^{A}\right)$ and the corresponding $C_{i}^{B}$ are multiplied, to obtain maps which represent the degree of fit between the two original maps $A$ and $B$ for each category:

$$
I_{j}=F_{j}^{A} \times C_{j}^{B} \quad j: 1, \ldots, r
$$

Figure 1 summarizes the procedure for obtaining each map $I_{j}$.

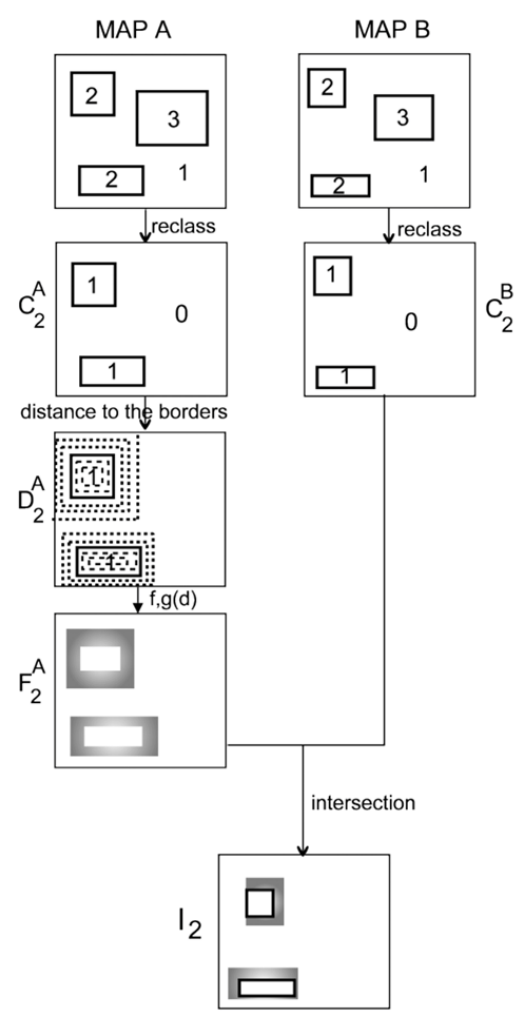

Fig. 1: Flowchart showing the proposed methodology for comparing maps 
5. Finally all of the previous maps $I_{j}$ are combined to form the final map of similarity between $B$ and $A$ :

$$
S_{A}^{B}=\cup I_{j} \quad j: 1, . ., r
$$

All the cells of $S_{A}^{B}$ take values between 0 and 1 , where 0 corresponds to a total absence of agreement between the original maps and where the value 1 corresponds to complete agreement.

6. Steps 1 to 5 are repeated taking map $B$ as reference, thus obtaining a final map of agreement between $A$ and $B$. Lastly, a map depicting the final similarity is constructed by determining, for each cell, the minimum value for the two maps of similarity that were calculated:

$$
S=\min \left(S_{A}^{B}, S_{B}^{A}\right)
$$

As well as the map of similarity which allows us to visually distinguish the similarity between the original maps, the degree of overall similarity can be summarized in a single value called a coefficient of similarity, which is given by the expression:

$$
S C=\frac{\sum_{i=1}^{N} S_{i}}{N}
$$

where $S_{i}$ represents the similarity values calculated for each cell $i$ of map $S$, and $N$ is the total number of cells in each image.

The coefficient of similarity does not consider similarity that may occur randomly, and this is why it is better to use another similarity measuring parameter known as the fuzzy kappa index. According to the definition of Hagen-Zanker et al. [6], this is given by the following expression:

$$
K_{f u z z y}=\frac{S C-E}{1-E}
$$

where $S$ and $E$ represent the calculated and expected similarity, respectively.

The expected similarity $E$ can be calculated using the Monte Carlo method, generating maps with random distributions of the categories with the same proportion of each category as the original maps.

\section{Study case}

To demonstrate how the proposed methodology works, we applied the method to two sets of maps. The first dataset was synthetic (illustrated in Figure 3) and was generated specifically to demonstrate how the proposed methodology works. The second dataset (Figure 3) consists of two temporal land-use maps of the same areas (with 7 categories) which correspond to the years 1985 and 1999.

In both examples the functions of category variation with distance were as follows:

$$
\begin{gathered}
f(d)=0.8 e^{-d} \\
g(d)=1-0.2 e^{-d}
\end{gathered}
$$

where $d$ is the distance from each cell to the nearest polygon boundary.

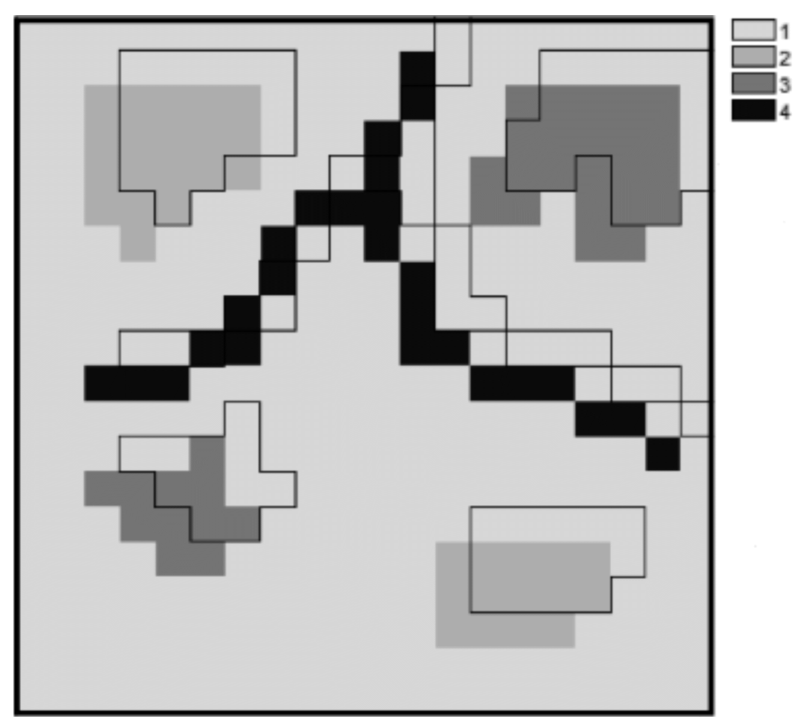

Fig. 2: Vector synthetic map B overlaying raster synthetic map A

In the synthetic dataset, map $B$ was obtained by translating the cells of the polygons of categories 2,3 and 4 in the northeastern direction by a distance equal to the diagonal length of each cell. In Figure 3, the boundaries of these categories have been superimposed on the respective categories in map $A$. If we did not take this translation into account (which one might assume to be due to a recording error) it is obvious that the match between the two maps would be total, and this is how it would be interpreted by an observer who visually analyzed the images separately. 


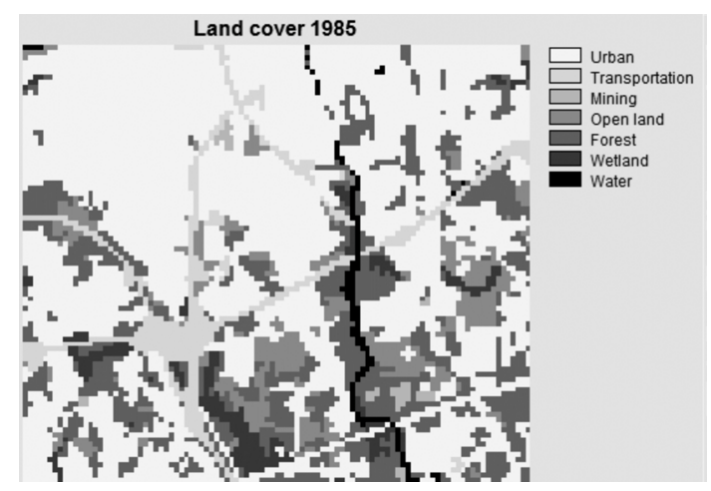

(a) Year 1985

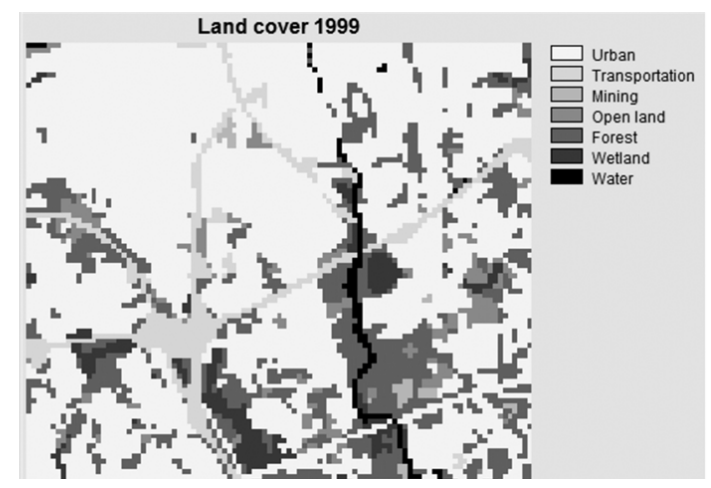

(b) Year 1999

Fig. 3: The two land-cover maps compared

\section{Results}

Comparison of the maps in the synthetic dataset gives the result shown in Figure 4, where shades of grey indicate similarity or degree of matching.

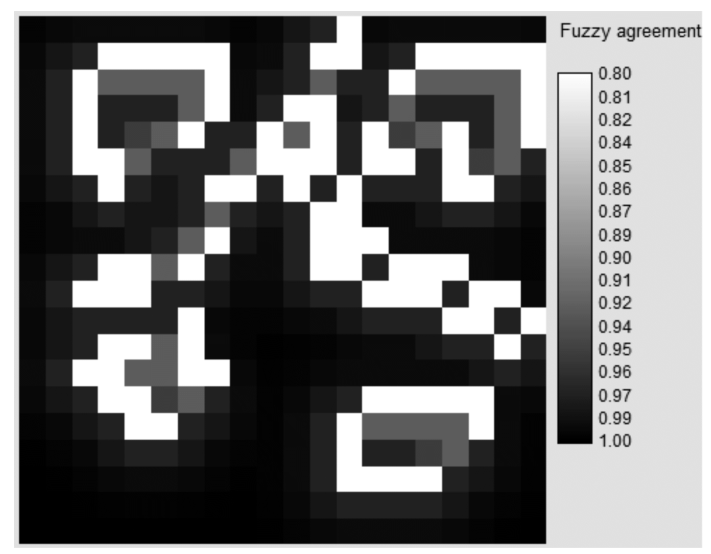

Fig. 4: Fuzzy agreement map for the two synthetic maps compared

Table 1 shows the values of the overall similarity index that were obtained with the proposed method together with the fuzzy kappa index, Boolean index and the coefficient of areal association (Taylor [13]). The fuzzy kappa index was obtained by a Monte Carlo simulation which generated 50 random maps with the same proportion of cells of each category as in map A and calculated the mean coefficient of similarity. Considering that the distribution patterns for the categories are the same in both maps, one would expect that the match would be very high, but as can be seen in Table 1, the Boolean kappa index is just 0.402 . In other words, according to this parameter the agreement between both maps is only $40 \%$ better than what would be obtained if we compared map A with another map in which the location of each cell of each category was determined randomly. It should be taken into account that the Boolean kappa index underestimates map agreement (Foody, [3]), and particularly in cases such as this one, in which there are only four classes and one of them-class 1 - prevails over the others. On the other hand, the fuzzy kappa index-calculated according to the definition given by Hagen-Zanker et al. [6] - is 0.756 , which is much more in line with the match that would be expected for two maps which have the same spacial patterns. The coefficient of areal association gave the highest value; this is only to be expected as this measure overestimates the agreement between maps because it does not account for chance agreement (Congalton et al. [1]).

As for the second dataset, Figure 5 shows the match between the two land-use maps. The lighter colours indicate a lower degree of agreement and so correspond to areas where there has been a change in land use between 1985 and 1999. In this example there are no errors due to erroneous recording, and so the differences between the two maps are basically due to variations in land use and to errors in the category assignment process. The greatest variations occurred in the central part of the area studied (coloured white in Figure 5), where urban land has encroached on open land and forest (see Figure 3). In Figure 5 the effect of introducing boundary uncertainty to the polygons can also be observed.

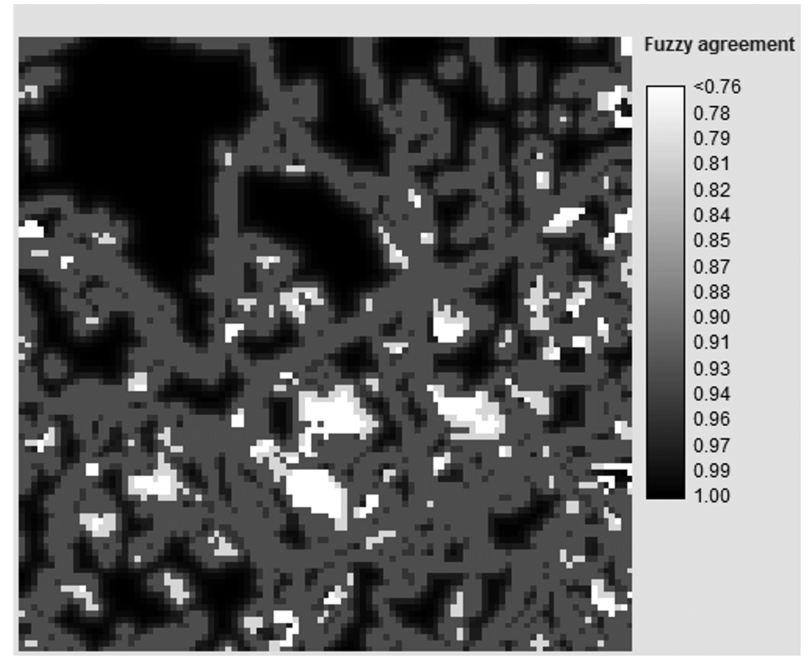

Fig. 5: Fuzzy agreement map for the two land-cover maps compared 
Table 1 shows the values for boolean kappa, the coefficient of areal association (CAA) and fuzzy kappa for this dataset. As can be seen, both are very similar and quite close to 1 , which indicates that overall there have been small changes over the years. As in the previous example, the kappa fuzzy index lies between the Boolean kappa and the coefficient of areal association. This is a positive feature, because, as was mentioned earlier, Boolean kappa tends to underestimate agreement, whereas the coefficient of areal association does the opposite.

Table 1: Comparison of coefficients of agreement between maps

\begin{tabular}{ccc}
\hline & Synthetic map & Land cover map \\
\hline Boolean kappa & 0.402 & 0.856 \\
CAA & 0.765 & 0.886 \\
Fuzzy kappa & 0.738 & 0.864 \\
\hline
\end{tabular}

\section{Conclusions}

In this article we describe a new methodology for comparing categorical raster maps that share the same legend. The method stems from the hypothesis that uncertainty in defining categories is greatest at the boundary between polygons.

Since maps are compared at the polygon level, this implies very high speed of calculation compared to methods that are based on comparing each cell with its neighbours. It also means a reduction in possible errors from erroneous registrations. Another advantage of this method is its simplicity, as it can be easily carried out in any GIS raster using common functions.

The results of the similarity analysis can be presented using the fuzzy kappa index. They can also be displayed graphically as a map of similarity where the match between the compared maps at each location is shown on a continuous scale as a value between 0 and 1 . The fact that the kappa fuzzy index lies between the Boolean kappa and the coefficient of areal association is positive, as Boolean kappa tends to underestimate agreement, whereas the coefficient of areal association tends to overestimate agreement.

\section{Acknowledgements}

This work is supported by project INCITE08PXIB304186PR of the Xunta de Galicia (Spain)

\section{References}

[1] R.L. Congalton, R. Oderwald, R.A. Mead, Assessing Landsat classification accuracy using discrete multivariate analysis statistical techniques, Photogram- metric Engineering and Remote Sensing, 167-1678 (1983).

[2] X.L. Dai, S. Khorram, Remotely sensed change detection based on artificial neural networks, Photogrammetric Engineering and Remote Sensing, 1187-1194 (1999).

[3] G.M. Foody, On the compensation for chance agreement in image classification accuracy assessment, Photogrammetric Engineering and Remote Sensing, 1459-1460 (1992).

[4] G.M. Foody, Status of land cover classification accuracy assessment, Remote Sensing of Environment, 185-201 (2002).

[5] S. Fritz, L. See, Comparison of land cover maps using fuzzy agreement, International Journal of Geographical Information Science, 787-807 (2005).

[6] A. Hagen-Zanker, B. Straatman, I. Uljee, Further developments of a fuzzy set map comparison approach, International Journal of Geographical Information Science, 769-785 (2005).

[7] Z. Ma, R.H. Redmond, Tau coefficients for accuracy assessment of classification of remote sensing data, Photogrammetric Engineering and Remote Sensing, 435-439 (1995).

[8] J.F. Mas, Monitoring land-cover changes: a comparison of change detection techniques, International Journal of Remote Sensing, 139-152 (1999).

[9] R.G. Pontius, Statistical methods to partition effects of quantity and location during comparison of categorical maps at multiple resolutions, Photogrammetric Engineering and Remote Sensing, 275-293 (2002).

[10] C. Power, A. Simms, R. White, Hierarchical fuzzy pattern matching for the regional comparison of land use maps, International Journal of Geographical Information Science, 77-100 (2001).

[11] G.H. Rosenfield, Fitzpatrick-Lins Coefficient of agreement as a measure of Thematic Classification accuracy, Photogrammetric Engineering and Remote Sensing, 131-137 (1986).

[12] A. Singh, Digital change detection using remotesensing data, International Journal of Remote Sensing, 989-1003 (1989).

[13] P. Taylor, Quantitative Methods in Geography (Boston: Houghton Mifflin Company, 1977).

[14] G.M. Wickware, P.J. Howarth, Procedures for change detection using Landsat digital data, International Journal of Remote Sensing, 277-291 (1981). 